\title{
Review
}

\section{Update on HER-2 as a target for cancer therapy Alternative strategies for targeting the epidermal growth factor system in cancer}

\author{
William J Gullick
}

Department of Biosciences, University of Kent at Canterbury, Canterbury, Kent, UK

Correspondence: William J Gullick, Department of Biosciences, University of Kent at Canterbury, Canterbury, Kent CT2 7NJ, UK.

Tel: +44 01227 823014; fax: +44 01227 827221; e-mail:W.J.Gullick@ukc.ac.uk

Received: 25 July 2001

Accepted: 11 September 2001

Published: 1 October 2001
Breast Cancer Res 2001, 3:390-394

(C) 2001 BioMed Central Ltd

(Print ISSN 1465-5411; Online ISSN 1465-542X)

\begin{abstract}
The epidermal growth factor (EGF) family of ligands and receptors interact to influence cell division, differentiation and motility. Much evidence supports their importance in causing and sustaining cell transformation in model systems and in human cancer. The exact mechanism by which this is achieved varies in different tumour types and from case to case. The EGF system is a target for new types of targeted chemotherapy. The choice of strategy will depend on the mechanism involved, however, and several approaches are under development or evaluation in clinical trials. Each will have a different spectrum of side effects and the potential for development of drug resistance.
\end{abstract}

Keywords: cancer, chemotherapy, growth factor, growth factor receptor

\section{Introduction}

The human body develops from a single cell into its final adult form, which contains roughly 100 million, million cells. In addition, many hundreds of millions of cells die and are replaced daily, particularly in epithelial tissues such as skin and the lining of the gastrointestinal tract. Traumatic or chronic injury also stimulates tissue repair, which involves much cell division. All of these processes are controlled, at least in part, by families of proteins called growth factors. These are usually small, membrane impermeable proteins with receptors that are consequently found, for at least part of their lifecycle, on the surface of responsive cells.

\section{Receptors}

The family of growth factors and receptors described in this series of reviews are known as the type 1 or EGF family. In simpler organisms such as worms or flies the family is a modest one, consisting of one receptor (Let 23) and one ligand (Lin 3) in Caenorhabditis elegans, and one receptor (DER) and four (Spitz, Gurken, Vein and Argos) or possibly five (including Keren, a very recently described gene) ligand genes in Drosophila melanogaster. Presumably as a consequence of the greater size and complexity of humans, this family has grown to consist of four receptor genes (EGFR/HER1, c-erbB2/HER2, c-erbB3/HER3 and c-erbB4/HER4).

Each receptor protein has the same basic structure, consisting of an extracellular amino-terminal domain, a single transmembrane spanning sequence and an intracellular cytoplasmic domain. This polypeptide chain is folded into domains with specific functions. The extracellular domain is divided into four regions based on two repeated sequences, termed L1 and L2, which are responsible for ligand recognition, and into further domains, termed S1 and $\mathrm{S} 2$, which are rich in cysteine residues and provide a framework on which to orientate the L1 regions. The transmembrane spanning sequence is far from the 'passive spacer' it was originally believed to be, providing inter-receptor con- 
tacts that are important in dimerization. The cytoplasmic domain contains three types of regions: the enzyme tyrosine kinase; sites of phosphorylation on tyrosine that allow interactions with intracellular second messenger systems (see next article by Monilola Olayioye 'Intracellular signaling pathways of ErbB2 and family members'); and sites of phosphorylation on serine and threonine residues that affect properties such as ligand affinity.

\section{Ligands}

The 10 characterized human ligand genes encode two distinct families that share a central ligand motif of approximately 50 amino acids, containing three disulphide bonds that stabilize the folded protein. The structure of this region has now been determined by X-ray crystallography [1] and by nuclear magnetic resonance [2]. The first group of ligands that share the property of binding directly to the EGF receptor are only produced in one form, but can be processed by proteolytic cleavage into several forms. The neuregulin genes (protein products of which bind to the cerbB3 and c-erbB4 receptors) produce a range of proteins through splicing of alternative exons, and these are further processed by glycosylation and proteolysis.

\section{Signalling}

Ligand binding induces receptor dimerization and oligomerization. It is not currently clear whether both are required for activating the cytoplasmic tyrosine kinase domain, but the end result is that the receptor becomes phosphorylated at specific sites in its own structure on the hydroxyl groups of tyrosine residues. A complex interplay of ligands in microenvironments induces selections of receptor homodimers and heterodimers in individual cells. Some combinations, particularly those including c-erbB2, are more effective at promoting cell division due to their greater affinity for ligands and their stability in the cell membrane, which maintains their ability to signal for a longer duration. Different receptors also have intrinsic abilities to select different second messengers because of the structure surrounding particular phosphorylation sites. The selection of second messengers may be further complicated by the use of different sites of phosphorylation in heterodimers than in homodimers.

This rather complicated system [3] plays important roles in development, wound healing and disease, notably in cancer. Ligands may act at a distance, carried in the bloodstream (endocrine); locally by diffusion (paracrine); by cell-to-cell contact (juxtacrine); and possibly within cells (intrakine). In natural settings, presumably the cell normally receives stimuli from most if not all of these routes. Some ligands, however, may be immobilized either on the surface of cells by their own transmembrane region or by other molecules such as heparan sulphate proteoglycans, with which they interact by weaker electrostatic forces and therefore can only act locally.

\section{Cancer}

During the more than 40 years of research carried out in EGF signalling, our understanding has greatly increased but there is clearly still a great deal to learn about its normal functions. Although fascinating in itself, this research has been further stimulated to an increasing degree by the appreciation of the critical importance of EGF signalling in cancer. Indeed, a significant amount of research conducted by pharmaceutical companies is now directed at exploiting this and closely related systems as targets for drug development.

Cancer cells grow at a rate faster than that at which they die; this is in contrast to cells in normal tissues, in which the rate is balanced or slightly in favour of cell death. It was originally hypothesized that cells cycled at their fastest rate unless they were restrained in some way, perhaps by nutritional or environment limitations, but also by specific factors collectively termed chalones. Despite much endeavour these remained elusive, sometimes with the suspicious characteristic of becoming less active as they became more pure. In the early 1960 s, however, Rita Levi-Montalcini purified nerve growth factor and Stanley Cohen (a PhD student in her laboratory) purified EGF [4]. This protein was originally termed 'tooth-lid factor', because the assay for its purification consisted of injecting fractions into newborn mice and measuring the time before their incisors erupted and their eyelids opened; this is not an assay commonly used today!

This, and similar data on purified molecules, strongly supported the concept that cells required positive stimuli to grow. Two observations were critical, fusing the field of growth factor research with that of cancer: the discovery that two retroviruses (simian sarcoma virus and avian erythroblastosis virus) contained a growth factor (plateletderived growth factor) $[5,6]$ and a mutationally activated growth factor receptor (EGF receptor), respectively, as critical oncogenes [7]. Introduction and expression of these proteins by the virus into susceptible animals or animal cells in culture led to cell transformation. Loss of expression or suppression of their activity made the cells less oncogenic, thereby providing the paradigm that these types of molecules may be responsible for the imbalance in growth that is observed in cancer.

In parallel, work was beginning on the analysis of growth factors and their receptors in human tumour specimens. Hendler and Ozanne [8] first showed, by immunocytochemical staining, that the EGF receptor was present at abnormal levels in human lung cancers. The development of antibodies that could detect expression of these receptors and their ligands in paraffin-embedded, formalin-fixed human tissues enabled larger series of cases to be examined [9]. Although being, in my view, far from complete in terms of accuracy, scale and coverage of molecular types, 
and certainly in terms of understanding of the information contained, some generalizations have been developed from this research.

Three mutually nonexclusive mechanisms lead to overactivity of growth factor receptors (Fig. 1). Receptors may be present in a normal form at a normal level, but be overactive because of unusually high amounts of ligands produced by a variety of mechanisms. First, through an indirect mechanism, mutations in genes such as Ras lead to increased expression of EGF-like ligands, but it appears that this may only augment cell transformation by Ras itself [10]. Other receptors, in particular G-protein-coupled receptors including gonadotrophin-releasing hormone receptor, caused increased activity of the ADAM family of metalloproteinases, which release active ligands from the cell surface by proteolysis [11]. Gene amplification has not reliably been reported as a mechanism for increased ligand expression (which is an interesting observation because amplification often causes receptor over-expression).

The second mechanism that causes over-activity of growth factor receptor signalling is over-expression of a normal growth factor receptor, either due to increased transcription or gene amplification, or both. It is not clear whether this requires the presence of some ligand or is sufficient to increase the amount of active receptor because of the equilibrium between monomer and dimers. This may be somewhat academic, however, because it is hard to conceive of a cell in an environment in which no ligands exist, but it could have relevance to the choice of and efficacy of different approaches to treatment.

Finally, growth factor receptors can be activated by point mutations (such as the Ret gene in multiple endocrine neoplasia-2A, an inherited predisposition to cancer) [12] and sporadically in the c-kit receptor (in gastrointestinal stromal tumours), but thus far there is no reliable evidence for this occurring in the type 1 receptor family. Deletion of various parts of the EGF receptor gene is quite commonly found in brain tumours, however [13]. The most frequent of these, called the type III mutant EGF receptor, involves the deletion of residues $6-273$ in the extracellular domain. This prevents ligand binding, but activates the receptor to approximately $10 \%$ of the level achieved by saturating ligand concentrations [14]. In this case the mutated gene is also amplified, suggesting that this level of activation does not achieve full transformation. Various reports have suggested that the type III receptor is expressed at very high prevalence in other tumour types, such as breast cancer. However, the EGFR gene is very rarely amplified in this type of disease and is even less frequently rearranged, so the underlying mechanism producing it must differ, possibly involving tumour specific alternative splicing. In our laboratory, however, we have not found evidence for the mutant receptor by polymerase chain reaction analysis in
Figure 1

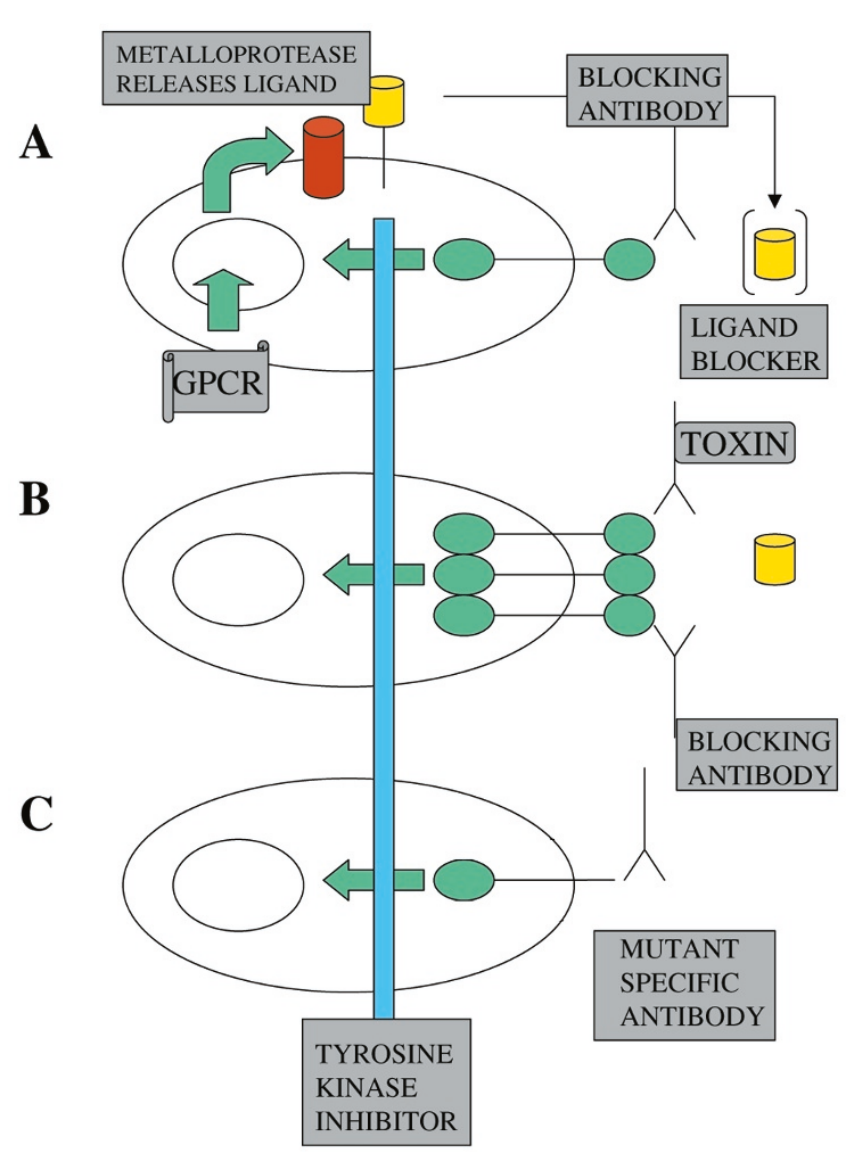

The epidermal growth factor family of ligands and receptors transform cells by different mechanisms. (A) A cell may express a normal level of receptors, but these are over-active because of the presence of excess levels of one or more ligands. The mechanisms by which excessive amounts of ligands are produced are not well established, but are not due to gene amplification. Some evidence suggests that other systems, such as G-protein-coupled receptors (GPCRs), may influence ligand processing by metalloproteases. (B) A cell may overexpress a receptor as a result of either gene amplification or increases in transcription, or both. (C) Some cancers, notably brain tumours, express mutant receptors that are partly constitutively active. The expression, processing and bioavailability of ligands can be targeted to suppress their actions. Receptors can be targeted by antibodies, which may inhibit ligand binding or have other activities, or by antibodies fused with toxic molecules or with small molecule tyrosine kinase inhibitors.

breast cancer cell lines, or by staining with a mutant receptor protein specific antibody in primary, fixed breast cancer. This issue is important to resolve, because it may be a very promising target for treatment [14].

\section{Treatment}

Different points in the ligand/receptor lifecycles represent targets for therapy (Fig. 1). Protease inhibitors can very effectively prevent the release of cell surface growth 
factors, but their effects may be rather promiscuous, suggesting that chronic use could have side effects. Inhibitors of G-proteins or G-protein-coupled receptors could suppress growth factor production in diseases such as prostate cancer, but clearly they may also have a more direct effect.

It is possible to treat patients with antibodies to growth factors or to induce immune responses to them, but it is likely that several growth factors would have to be neutralized for this to have significant effect. Other compounds, such as suramin, mimic to some degree the proteoglycans that naturally sequester growth factors. Clearly, there are opportunities to develop more specific, less toxic growth factor neutralizing molecules. None of these strategies would a priori be likely to affect the growth of cancers expressing mutated receptors that have ligand independent activity.

The second strategy is to prevent receptor signalling more directly. Antibodies have been made that inhibit ligand binding and have been shown to generate responses in patients [15]. Other antibodies such as Herceptin bind to the extracellular domain of receptors in the same way but, because their target c-erbB2 has no known directing ligand, they presumably act by other mechanisms [16] In the former case, the ligand blocking antibodies may be effective in cells with normal or over-expressed receptors as long as abundant ligand is present. Over-expressed receptors could be a target for ligand or antibody linked toxins, but paradoxically these are apparently so toxic that normal tissues were also affected, resulting in unacceptable toxicity [17]. Mutant receptors can be targeted with specific antibodies with or without cytotoxic functions because they should be highly specific in their actions [14].

Finally, because excess receptor signalling is the outcome in all of the examples discussed, inhibition of tyrosine kinase activity using orally available small synthetic molecules may be the most widely applicable strategy. These types of molecules may be required to be targeted very specifically, because it is now clear that activation of molecules such as c-erbB4 has antiproliferative effects [18] but it is not clear how important this system is in cancer cells.

Although these approaches look very promising, there are clearly some confounding issues, the most important being side effects and the development of drug resistance. Side effects may be on-target (i.e. by affecting the receptor itself), off-target (i.e. via related proteins such as other kinases), or nonspecific. They may be mediated via the drug itself or by its metabolites. They may be acute or chronic, reversible or irreversible. A common profile of acute effects appears to be shared by two EGF receptor antibodies and by tyrosine kinase inhibitors: acute acniform rash and diarrhoea, which resolve after withdrawal of the drug.
The development of drug resistance is almost inevitable. How long it will take to develop and what alternative pathways will become dominant are at present unpredictable. If these pathways can be identified then it may be possible to develop further drugs directed at them and patients may be transferred to these if their cancer progresses.

\section{Conclusion}

The EGF family is involved through a variety of mechanisms in the majority of epithelial cancers. Diagnosis of these mechanisms in different tumour types and in individual patients will provide the rationale for clinical trials and suggest the appropriate choice of drug. Undesirable side effects and drug resistance may be minimized by the use of different drugs used in combination or sequentially. The encouraging prospect is that patients with breast and other types of cancer, and the clinicians who treat them, will have more effective, less toxic drugs available in the relatively near future.

\section{References}

1. Lu H-S, Chai J-J, Huang B-R, He C-H, Bi R-C: Crystal structure of the human epidermal growth factor and its dimerisation. $J$ Biol Chem 2001, 276:34913-34917.

2. Cooke RM, Wilkinson AJ, Baron M, Pastore A, Tappin MJ, Campbell ID, Gregory H, Sheard: The solution structure of human epidermal growth factor. Nature 1987, 327:339-341.

3. Gullick WJ: The type 1 growth factor receptors and their ligands considered as a complex system. Endocr Relat Cancer 2001, 8:75-82.

4. Cohen S: EGF and its receptor: historical perspective. $J$ Mammary Gland Biol Neoplasia 1997, 2:93-96.

5. Deuel TF, Huang JS, Huang SS, Stroobant P, Waterfield MD: Expression of a platelet-derived growth factor-like protein in simian sarcoma virus transformed cells. Science 1983, 21: 1348-1350.

6. Robbins KC, Antoniades HN, Devare SG, Hunkapiller MW, Aaronson SA: Structural and immunological similarities between simian sarcoma virus gene product(s) and human plateletderived growth factor. Nature 1983, 305:605-608.

7. Downward J, Yarden Y, Mayes E, Scrace G, Totty N, Stockwell P, Ullrich A, Schlessinger J, Waterfield MD: Close similarity of epidermal growth factor receptor and v-erb-B oncogene protein sequences. Nature 1984, 307:521-527.

8. Hendler FJ, Ozanne BW: Human squamous cell lung cancers express increased epidermal growth factor receptors. $J$ Clin Invest 1984, 74:647-651.

9. Srinivasan R, Benton E, McCormick F, Thomas H, Gullick WJ: Expression of the c-erbB-3/HER-3 and c-erbB-4/HER-4 growth factor receptors and their ligands, neuregulin-1 alpha, neuregulin-1 beta, and betacellulin, in normal endometrium and endometrial cancer. Clin Cancer Res 1999, 5:2877-2883.

10. McKay IA, Malone P, Marshall CJ, Hall A: Malignant transformation of murine fibroblasts by a human c-Ha-ras-1 oncogene does not require a functional epidermal growth factor receptor. Mol Cell Biol 1986, 6:3382-3387.

11. Prenzel N, Fischer OM, Streit S, Hart S, Ullrich A: The epidermal growth factor receptor family as a central element for cellular signal transduction and diversification. Endocr Relat Cancer 2001, 8:11-31.

12. Lohmeyer M, Gullick WJ. Mutational activation of receptor tyrosine kinases. In: Hormones and Growth Factors in Development and Neoplasia. Edited by Dickson RB, Salomon DS. New York: John Wiley and Sons, Inc.; 1998:289-309.

13. Kuan CT, Wikstrand CJ, Bigner DD: EGF mutant receptor vIII as a molecular target in cancer therapy. Endocr Relat Cancer 2001, 8:83-96.

14. Hills D, Rowlinson-Busza G, Gullick WJ: Specific targeting of a mutant, activated EGF receptor found in glioblastoma using a monoclonal antibody. Int J Cancer 1995, 63:537-543. 
15. Mendelsohn J, Baselga J: The EGF receptor family as targets for cancer therapy. Oncogene 2000, 19:6550-6565.

16. Molina MA, Codony-Servat J, Albanell J, Rojo F, Arribas J, Baselga $\mathrm{J}$ : Trastuzumab (herceptin), a humanized anti-Her2 receptor monoclonal antibody, inhibits basal and activated Her2 ectodomain cleavage in breast cancer cells. Cancer Res 2001, 61:4744-4749.

17. Pastan II, Kreitman RJ: Immunotoxins for targeted cancer therapy. Adv Drug Deliv Rev 1998, 31:53-88.

18. Sartor Cl, Zhou H, Kozlowska E, Guttridge K, Kawata E, Caskey L, Harrelson J, Hynes N, Ethier S, Calvo B, Earp HS: Her4 mediates ligand-dependent antiproliferative and differentiation responses in human breast cancer cells. Mol Cell Biol 2001, 21:4265-4275. 